\title{
FLAT ANALYTIC EXTENSIONS
}

BY

\author{
ANA M. D. VIOLA-PRIOLI( $\left.{ }^{1}\right)$
}

\begin{abstract}
This paper is concerned, in the first place, with the conditions to be imposed on an ideal $I$ of the power series ring in one indeterminate $A[[x]]$ ( $A$ noetherian) in order that the analytic extension $B=A[[x]] / I$ be a flat $A$-module.

Also the relationship between the projectivity and finiteness of $B$ is found when the content of $I$ (the ideal of $A$ generated by the coefficients of all power series in $I$ ) equals $A$. A generalization of this result to the power series ring in any finite number of indeterminates is obtained when $A$ is local, noetherian of Krull $\operatorname{dim} \geqslant 1$, and under certain restrictions on $I$, for the global case but only for domains.

Finally, a contribution to the problem of the finiteness of $I$ when $A[[x]] / I$ is a flat analytic extension is given for $A$ a local ring, not necessarily noetherian.
\end{abstract}

Introduction. A polynomial extension of a commutative ring $A$ is defined as a quotient $A[x] / I$ where $I$ is an $A[x]$-ideal. The flatness and projectivity of such extensions as $A$-modules and the finiteness of $I$ have been considered by M. Nagata [7], J. Ohm and W. Heinzer [9] , J. Ohm and D. Rush [8], W. Vasconcelos [12] and [13], and others.

In this paper we are going to study similar problems for the case of an analytic extension of a commutative ring $A$, i.e., a quotient $A[[x]] / I$, where $I$ is an ideal of the power series ring $A[[x]]$.

The results obtained resemble the corresponding ones for polynomial extensions, but technical difficulties arise as we cannot reduce to the local case the way it is done for polynomial extensions since localization does not commute with infinite direct products-which is the module structure in question. This considerably reduces the available techniques to deal with the problem. Another source of difficulty is that, except in the noetherian case, we cannot reduce the question to lower dimensional rings because $A / J[[x]] \simeq A[[x]] \otimes A / J$ is not

Received by the editors November 26, 1973.

AMS (MOS) subject classifications (1970). Primary 13B99; Secondary $13 \mathrm{~J} 05$.

Key words and phrases. Power series, flat module, projective module, content, invertible ideal, pure submodule, Krull dimension, coherency.

(1) This paper is part of the author's Ph.D. dissertation, written under the direction of Professor Wolmer Vasconcelos at Rutgers, The State University. 
always true. That is why our most precise results will involve noetherian local rings. Also the coherence of a noetherian ring $A$ assures the flatness of the power series ring $A[[x]]$ as $A$-module, a fact we will use often.

A summary of the content of this paper is given next:

$\S 1$ is devoted to setting notations and basic concepts.

In $\S \S 2$ and 3 we extend Vasconcelos' result in [12] to flat analytic extensions in the following manner:

Theorem 3.4. Let $A$ be a noetherian ring and $I$ an $A[[x]]$-ideal. Then $B=A[[x]] / I$ is a flat analytic extension of $A$ if and only if $I$ is a projective ideal of $A[[x]]$ and $c(I)$ (the so-called content of $I$ )-the ideal of $A$ generated by the coefficients of all power series in I-is generated by an idempotent element of $A$. Moreover, if $c(I)=A$, then $B$ is A-projective if and only if $B$ is a finitely generated flat A-module.

$\S 4$ is concerned with the generalization of the above result for power series rings in any finite number of indeterminates. We prove the following theorems.

Theorem 4.6. Let $A$ be a noetherian domain with Krull dimension $A \geqslant$ 1 and $I$ an $A\left[\left[x_{1}, \cdots, x_{n}\right]\right]$-ideal. If $B=A\left[\left[x_{1}, \cdots, x_{n}\right]\right] / I$ is A-projective and $I \cap A\left[\left[x_{i}\right]\right] \neq 0$ for at least $n-1 x_{i}^{\prime}$ 's, then $B$ is a finitely generated A-module.

When Krull $\operatorname{dim} A=0$ (i.e., when $A$ is artinian) the question is not relevant as projective and flat modules coincide.

Theorem 4.7. Let $A$ be a local noetherian ring with $\operatorname{Krull} \operatorname{dim} A \geqslant 1$ and $I$ an $A\left[\left[x_{1}, \cdots, x_{n}\right]\right]$-ideal. Then $B=A\left[\left[x_{1}, \cdots, x_{n}\right]\right] / I$ is $A$-projective if and only if it is a finitely generated flat A-module.

In $\S 5$ we present two contributions to the question of the finiteness of $I$ when $A[[x]] / I$ is a flat analytic extension and $A$ a local ring. The hypothesis of noetherianness is no longer needed in this section.

COROLlaRy 5.5. Let $A$ be a local ring, I a finitely generated $A[[x]]$ ideal, and $A[[x]] / I$ a flat analytic extension of $A$. If there exists a power series $f \in I$ with $c(f)$ finitely generated, then $I$ is principal.

TheOREM 5.6. Let $A$ be a local ring with $\mathrm{Krull} \operatorname{dim} A=0, I$ an $A[[x]]$-ideal, and $A[[x]] / I$ a flat analytic extension of $A$. If $c(I)=A$, then $I$ is principal.

This paper is based on the author's doctoral dissertation at Rutgers, The State University. The author wishes to express her deepest gratitude to her thesis adviser, Professor Wolmer Vasconcelos, for his generous guidance, help 
and encouragement in the preparation of this paper.

1. Preliminaries. Throughout, $A$ will denote a commutative ring with identity element, usually written 1 , and all modules will be unitary. For terminology, notation and basic results we will use [1] or [4] .

We begin by recalling some elementary notation and, for easy reference, some results to be used in the text.

Whenever $A$ is a domain with field of quotients $K, \bar{A}$ will stand for the integral closure of $A$ in $K$.

An $A$-algebra $B$ will be called finite or $A$-finite if it is finitely generated as $A$-module.

We say that a ring $A$ is connected if its spectrum is a connected topological space, or equivalently, if it has no idempotents distinct from the identity or 0 .

An $A$-module is said to be reduced if it has no nonzero divisible submodules.

Given $E$ and $F, A$-modules, and $\gamma: E \rightarrow F$ a monomorphism we say that $\gamma$ is pure or that $E$ is pure in $F$ if for every $A$-module $L$

$$
0 \rightarrow E \otimes_{A} L \stackrel{\bar{y}}{\longrightarrow} F \otimes_{A} L, \quad \bar{\gamma}=\gamma \otimes 1_{L},
$$

is exact, or equivalently if every system of equations $e_{i}=\sum_{j=1}^{m} a_{i j} x_{j}, 1 \leqslant i \leqslant n$, with $e_{i} \in E$ and $a_{i j} \in A$ having a solution in $F$ also has a solution in $E$. (See [1, Chapter I, §2, Proposition 13].)

One notes that if $0 \rightarrow M \rightarrow N \rightarrow L \rightarrow 0$ is exact and $L$ is flat, then $M$ is pure in $L$, and hence for every $A$-module $H, 0 \rightarrow M \otimes_{A} H \rightarrow N \otimes_{A} H$ $\rightarrow L \otimes_{A} H \rightarrow 0$ is exact.

Also, if $0 \rightarrow M \rightarrow N \rightarrow L \rightarrow 0$ is an exact sequence of $A$-modules with $M$ and $N$ flat and $M$ pure in $N$, then also $L$ is flat.

The following is a known fact that we will use frequently.

Proposition 1.1. Let $A$ be a noetherian ring and $E \rightarrow F$ a monomorphism of A-modules with $F$ flat. Then $E$ is pure in $F$ if and only if, for every prime ideal $P, 0 \rightarrow E \otimes_{A} A / P \rightarrow F \otimes_{A} A / P$ is exact.

Proof. One direction is trivial. As for the other one, let $M$ be any module; then $M$ may be written as $M=\operatorname{liminj} M_{i}$ finitely generated $A$-modules. Since direct limit is an exact functor and it commutes with tensor product, we may assume that $M$ is finitely generated. We may apply [4, Exercise 7, p. 53] to obtain $A$-modules $M_{i}$ such that $0=M_{0} \subset M_{1} \subset \cdots \subset M_{n-1} \subset M_{n}=M$ with $M_{i} / M_{i-1}=A / P_{i}$ and $P_{i}$ prime ideals, for $1 \leqslant i \leqslant n$.

Assuming $n=2$ (and then proceeding by induction) we have $0 \rightarrow M_{1}$ $\rightarrow M \rightarrow A / P \rightarrow 0$, with $P$ prime. Tensor with $E$ and $F$ to get 


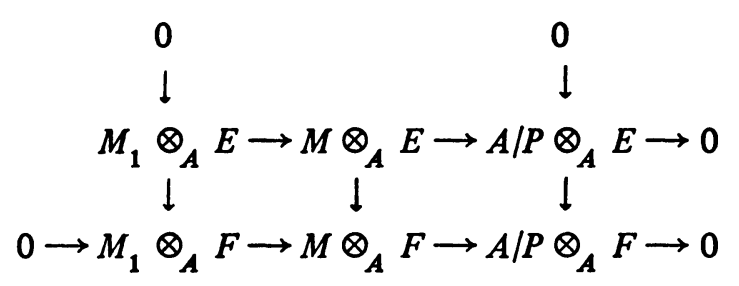

Chasing the diagram we see that $0 \rightarrow M \otimes_{A} E \rightarrow M \otimes_{A} F$ is exact. Q.E.D.

If $B$ is a projective $A$-module, for each prime ideal $P, B_{P}$, the localization of $B$ with respect to $A \backslash P$ is by [5] a free $A_{P}$-module with basis of cardinality $r_{B}(P)$. Notice that when $B$ is finite, $r_{B}(P)$ defines a map $r_{B}$ : $\operatorname{Spec}(A) \rightarrow \mathrm{Z}$ known as the rank function of $B$. One can see that if $m$ is a maximal ideal, then $r_{B}(m)=[B / m B: A / m]$.

Proposition 1.2. Let $A$ be a domain and $B$ a projective A-module. Assume there is a prime ideal $Q$ such that $r_{B}(Q)=n<\infty$. Then $r_{B}(P)=n$ for every prime ideal $P$ of $A$.

Proof. By the definition of rank, $B_{Q}=\left(A_{Q}\right)^{n}$. Assume that $r_{B}(P)=\lambda$ for some prime $P$, i.e., $B_{P}=\left(A_{P}\right)^{\lambda}$. If $K$ is the field of quotients of $A$, one has

$$
\begin{aligned}
K^{\lambda} & \simeq\left(A_{P}\right)^{\lambda} \otimes_{A_{P}} K \simeq B_{P} \otimes_{A_{P}} K \simeq\left(B \otimes_{A} A_{P}\right) \otimes_{A_{P}} K \simeq B \otimes_{A} K \\
& \simeq\left(B \otimes_{A} A_{Q}\right) \otimes_{A_{Q}} K \simeq B_{Q} \otimes_{A_{q}} K \simeq\left(A_{Q}\right)^{n} \otimes_{A_{Q}} K \simeq K^{n},
\end{aligned}
$$

which implies $\lambda=n$. Q.E.D.

If $A$ is a ring and $x_{1}, x_{2}, \cdots, x_{n}$ are indeterminates, we will denote by $A\left[\left[x_{1}, \cdots, x_{n}\right]\right]$ the ring of power series in $x_{1}, \cdots, x_{n}$ with coefficients in $A$. We make here a number of elementary remarks on this ring. An element $f\left(x_{1}, \cdots, x_{n}\right) \in A\left[\left[x_{1}, \cdots, x_{n}\right]\right]$ is a unit if and only if $f(0, \cdots, 0)$ is a unit in $A$. Consequently, every power series over a field with nonzero constant term is a unit. It is also easy to verify that an ideal $M$ of $A\left[\left[x_{1}, \cdots, x_{n}\right]\right]$ is maximal if and only if

$$
M=m A\left[\left[x_{1}, \cdots, x_{n}\right]\right]+\sum_{i=1}^{n} x_{i} A\left[\left[x_{1}, \cdots, x_{n}\right]\right]
$$

with $m$ maximal in $A$, uniquely determined.

We note that if $J$ is an ideal of $A$ and if $J\left[\left[x_{1}, \cdots, x_{n}\right]\right]$ is the ideal of $A\left[\left[x_{1}, \cdots, x_{n}\right]\right]$ whose elements are power series with coefficients in $J$, then in general, $J\left[\left[x_{1}, \cdots, x_{n}\right]\right] \supsetneqq J A\left[\left[x_{1}, \cdots, x_{n}\right]\right]$. However, if $J$ is finitely generated they coincide and then 


$$
A\left[\left[x_{1}, \cdots, x_{n}\right]\right] \otimes A / J \simeq A / J\left[\left[x_{1}, \cdots, x_{n}\right]\right],
$$

a remark we shall use repeatedly.

If $f\left(x_{1}, \cdots, x_{n}\right)-A\left[\left[x_{1}, \cdots, x_{n}\right]\right]$ we will refer to it indistinctly as either $f\left(x_{1}, \cdots, x_{n}\right)$ or $f$.

Given a power series $f, c_{A}(f)$ (or merely $c(f)$ if the ring $A$ is clear) will denote the content of $f$, i.e., the ideal of $A$ generated by the coefficients of $f$. Note that if $a \in A$ and $f \in A\left[\left[x_{1}, \cdots, x_{n}\right]\right]$, then $c(a f)=a c(f)$. If $I$ is an ideal of $A\left[\left[x_{1}, \cdots, x_{n}\right]\right], c(I)$ will stand for the ideal of $A$ generated by the coefficients of all power series in $I$.

Let us recall that if $k$ is a field, then $k[[x]]$ is a principal ideal domain. Also note that if $f_{i}\left(x_{i}\right) \neq 0$ are power series in $k\left[\left[x_{i}\right]\right]$, then

$$
k\left[\left[x_{1}, \cdots, x_{n}\right]\right] /\left(f_{1}\left(x_{1}\right), \cdots, f_{n}\left(x_{n}\right)\right)
$$

is a finite-dimensional space over $k$.

2. Characterization of flat analytic extensions. Given an ideal $I$ of $A[[x]]$, consider the following exact sequence of $A$-modules

$$
0 \rightarrow I \rightarrow A[[x]] \rightarrow B \rightarrow 0 .
$$

$B$ will be called an analytic extension of $A$.

Our first aim is to find necessary and sufficient conditions on $I$ for $B$ to be a flat analytic extension of a noetherian ring $A$. We will make use of the following lemmas.

LEMMA 2.1. Let $A$ be any ring and $0 \neq f(x)=\Sigma_{i=0}^{\infty} a_{i} x^{i}$. Assume that there exists $n$ such that $a_{n}$ is a non-zero-divisor of $A$. Let $g(x)=\sum_{i=0}^{\infty} b_{i} x^{i}$ with $c(g)$ finitely generated. If $f(x) g(x)=0$, then $g=0$.

Proof. Call $s=a_{n}$. Since $s$ is a non-zero-divisor, $A \subset A_{s}$ ( $A_{s}=$ ring of fractions with respect to the multiplicative set of powers of $s$ ), and therefore there exists an injective homomorphism $A[[x]] \rightarrow A_{s}[[x]]$. Hence, $f(x) g(x)=0$ in $A_{s}[[x]]$ also, and we may assume that $a_{n}=1 ; f(x) g(x)=0$ translates into the following system of equations:

$$
\begin{aligned}
a_{0} b_{0} & =0, \\
a_{1} b_{0}+a_{0} b_{1} & =0, \\
& \vdots \\
a_{r} b_{0}+a_{r-1} b_{1}+\cdots+a_{0} b_{r} & =0, \\
1 b_{0}+a_{n-1} b_{1}+\cdots+a_{0} b_{n} & =0,
\end{aligned}
$$


Let $m$ be a maximal ideal of $A$ and $I=c(g)$. We will show that $I m=$ I. Assume that $a_{r}$ is the first coefficient of $f$ not in $m$. (It exists by the choice of $s$.) Thus

$$
a_{r} b_{0}=-a_{r-1} b_{1}-\cdots-a_{0} b_{r} \in m I .
$$

Since $m$ is maximal, we have

$$
1=d+a a_{r} \text { with } d \in m \text { and } a \in A .
$$

Hence $b_{0}=b_{0} d+a b_{0} a_{r} \in m I$. Also by the election of $r$

$$
a_{r} b_{1}=-a_{r+1} b_{0}-a_{r-1} b_{2}-\cdots-a_{0} b_{r+1} \in m I
$$

and

$$
b_{1}=b_{1} d+a a_{r} b_{1} \in m I \text {. }
$$

Repeat the procedure to get $b_{i} \in m I$ for every $i$, which implies $I=m I$ for each maximal $m$ of $A$. It follows from Nakayama's lemma that $I=0$ and hence $g=0$. Q.E.D.

Lemma 2.2. Let $A$ be any ring, $I$ an ideal of $A[[x]]$ with $c(I)=A$. Then there exists a power series $g(x) \in I$ such that $b_{n}=1$ for some coefficient $b_{n}$ of $g$.

Proof. Since $c(I)=A$, there exist $a_{i n_{i}}$, coefficients of $f_{i}(x)=$ $\sum_{i=0}^{\infty} a_{i} x^{j} \in I$, and $r_{i} \in A$ such that

$$
1=\sum_{i=1}^{s} r_{i} a_{i n_{i}}
$$

Without loss of generality we may assume that $n_{1} \leqslant n_{2} \leqslant \cdots \leqslant n_{s}$. It follows that the power series

$$
g(x)=r_{1} f_{1} x^{n_{s}-n_{1}}+\cdots+r_{s-1} f_{s-1} x^{n_{s}-n_{s-1}}+r_{s} f_{s} \in I
$$

and its $n_{s}$ th coefficient is 1 by (2). Q.E.D.

LEMMA 2.3. Let $A$ be a noetherian ring and consider the exact sequence of $A$-modules in (1) with $I$ projective. Then $\operatorname{Tor}_{1}^{A}(B, A / P)=0$ for every prime ideal $P$, if and only if $\operatorname{Tor}_{1}^{A}(B, A / m)=0$ for each maximal ideal $m$.

Proof. Let us reason by contradiction. Among all prime ideals $Q$ such that $\operatorname{Tor}_{1}^{A}(B, A / Q) \neq 0$, pick $P$ a maximal such. Note that $P$ is not a maximal ideal. Take $a \in P$ and consider the following exact sequence

$$
0 \rightarrow A / P \stackrel{\bar{a}}{\longrightarrow} A / P \rightarrow A /(P, a) \rightarrow 0,
$$

where $\bar{a}$ is the image of $a$ in $A / P$. 
Tensoring with $B$ we get

$$
\begin{aligned}
& \cdots \rightarrow \operatorname{Tor}_{2}^{A}(A /(P, a), B) \rightarrow \operatorname{Tor}_{1}^{A}(A / P, B) \\
& \bar{a} \\
& \stackrel{T_{o r}}{A}(A / P, B) \rightarrow \operatorname{Tor}_{1}^{A}(A /(P, a), B) \rightarrow \cdots .
\end{aligned}
$$

Since $A[[x]]$ is $A$-flat and $I$ is $A[[x]]$-projective, $I$ is also a flat $A$-module. It follows then that flat $\operatorname{dim}_{A} B \leqslant 1$ and thus $\operatorname{Tor}_{2}^{A}(A /(P, a), B)$ $=0$. We claim that, by the election of $P, \operatorname{Tor}_{1}^{A}(A /(P, a), B)=0$. In fact, if we call $M=A /(P, a)$, by $[4$, Exercise 7, p. 53] , we have a composition series

$$
0 \subset M_{1} \subset M_{2} \subset \cdots \subset M_{n-1} \subset M_{n}=M
$$

where $M_{i} / M_{i-1} \simeq A / Q_{i}$ and the $Q_{i}$ 's are prime ideals of $A$. Note that $P \varsubsetneqq$ $Q_{i}$, so by the choice of $P$

$$
0=\operatorname{Tor}_{1}^{A}\left(A / Q_{i} B\right)=\operatorname{Tor}_{1}^{A}\left(M_{i} / M_{i-1}, B\right) \text { for all } i .
$$

We obtain then $\operatorname{Tor}_{1}^{A}(A / P, B) \simeq \operatorname{Tor}_{1}^{A}(A / P, B)$ via $\bar{a}$, which implies that $\operatorname{Tor}_{1}^{A}(A / P, B)$ is divisible by any $0 \neq \bar{a} \in A / P$. If we now tensor (1) with $A / P$, we get the following exact sequence:

$$
0 \rightarrow \operatorname{Tor}_{1}^{A}(A / P, B) \rightarrow I / P I \rightarrow A / P[[x]] \rightarrow B / P B \rightarrow 0 .
$$

Therefore, $I / P I$ admits a nonzero submodule which is divisible by every nonzero element of $A / P$. But this is impossible because, say, $I / P I$ is projective over $A / P[[x]]$. Thus $A / P$ is a field, which is a contradiction. Q.E.D.

TheOREM 2.4. Let $A$ be a noetherian ring and $B$ as in (1). Then $B$ is a flat anaiytic extension of $A$ if and only if $I$ is a projective ideal of $A[[x]]$ and $c(I)$ is generated by an idempotent element of $A$.

Proof. Assume that $B$ is flat and $I \neq 0$. Let $J=c(I)$ and tensor (1) with $A / J$ to get

$$
0 \rightarrow I / J I \rightarrow A / J[[x]] \rightarrow B / J B \rightarrow 0 .
$$

Since $I \subset J A[[x]]$ by the definition of content, we have $I=J I$ and hence $J=J^{2}$. This implies that $J=A e$, with $e$ idempotent, and the second assertion is proved.

For the first one we will assume that $c(I)=A$, as otherwise we decompose $A=A e \times A(1-e)$.

Let $M$ be a maximal ideal of $A[[x]]$ and consider $m=M \cap A$. Tensor (1) with $A / m$ to get

$$
0 \rightarrow I / m I \rightarrow A / m[[x]] \rightarrow B / m B \rightarrow 0 .
$$

$A / m[[x]]$ is a power series ring over a field, and hence a principal ideal 
domain. So $I / m I$ is a principal ideal of $A / m[[x]]$ and thus there exists $f \in$ $I$ such that $I=f A[[x]]+m A[[x]] \cap I$. But since $B$ is a flat module we have $m A[[x]] \cap I=\operatorname{Im} A[[x]]$ and thus

$$
I=f(x) A[[x]]+m A[[x]] I .
$$

Localize at $M$ to get

$$
I_{M}=f(x) A[[x]]_{M}+m A[[x]]_{M} I_{M},
$$

which by Nakayama's lemma implies $I_{M}=f(x) \cdot A[[x]]_{M}$, i.e., $I$ is locally principal. Also by Lemmas 2.1 and 2.2, $I$ contains a non-zero-divisor; so $f$ too is a non-zero-divisor, and therefore $I$ is projective.

Conversely, let us assume that $I$ is a projective ideal and reduce to the case when $c(I)=A$. To see that $B$ is flat (by Proposition 1.1 and Lemma 2.3) it is enough to show that $\operatorname{Tor}_{1}^{A}(B, A / m)=0$ for any maximal ideal $m$ of $A$.

By tensoring (1) with $A / m$ we obtain

$$
0 \rightarrow \operatorname{Tor}_{1}^{A}(B, A / m) \rightarrow I / m I \stackrel{\lambda}{\longrightarrow} A / m[[x]] \rightarrow B / m B \rightarrow 0 .
$$

If $M=(m, x), I_{M}$ is a principal $A[[x]]_{M}$-module. It follows that $I_{M} \otimes_{A[[x]]_{M}} A / m[[x]] \simeq I / m I$ is a principal $A / m[[x]]$-module, i.e. $I / m I=$ $[f] A / m[[x]]$ where $[f]$ is the image of $f$ in $I / m I$. Call $\bar{f}=\lambda[f]$. Since $c(I)=A, f$ cannot have all its coefficients in $m$, and hence $\bar{f} \neq 0$. Therefore, the endomorphism of $A / m[[x]]$ induced by multiplication by $\bar{f}$ is a monomorphism, i.e., the following is an exact sequence

$$
0 \rightarrow A / m[[x]] \stackrel{\bar{f}}{\longrightarrow} A / m[[x]] \rightarrow B / m B \rightarrow 0
$$

and it follows that $\operatorname{Tor}_{1}^{A}(B, A / m)=0$. Q.E.D.

3. Projectivity of analytic extensions. Given the exact sequence (1) with $A$ noetherian and $B$ projective, we want to see under what conditions $B$ is finite. If $A$ is not connected, it can be expressed as $A=\prod_{i=1}^{n} A e_{i}$ with $e_{i}$ idempotents and $A e_{i}$ connected for all $i$. Therefore it will be enough to consider the problem in each factor, and hence we may assume first that $A$ is connected.

THEOREM 3.1. Let $A$ be a connected noetherian ring and consider the exact sequence (1) with $B$ projective A-module. Then if $I \neq 0, B$ is $A$-finite and if $I=0, A$ is artinian.

Proof. Let us assume $I \neq 0$. By Theorem 2.4 and since $A$ is connected we have $c(I)=A$. By Lemma 2.2 there exists $f(x)=\Sigma_{i=0}^{\infty} a_{i} x^{i} \in I$ such that $a_{i}=1$ for some index $i$. 
Let $m$ be any maximal ideal of $A$, and let us assume that $n$ is minimal with the property that $a_{n} \notin m$. Tensor (1) with $A / m$ to get

$$
0 \rightarrow I / m I \rightarrow A / m[[x]] \rightarrow B / m B \rightarrow 0 \text {. }
$$

If we denote with $\bar{a}$ the image of $a$ in $A / m$, then

$$
\bar{f}(x)=\bar{a}_{n} x^{n}+\bar{a}_{n+1} x^{n+1}+\cdots=x^{n}\left(\bar{a}_{n}+\bar{a}_{n+1} x+\cdots\right) \in I / m I .
$$

But $\bar{a}_{n}+\bar{a}_{n+1} x+\cdots$ is invertible in $A / m[[x]]$ for $\bar{a}_{n} \neq 0$, therefore

$$
B / m B=\frac{A / m[[x]] /\left(x^{n}\right)}{I / m I /\left(x^{n}\right)}
$$

is a finite $A / m$-module and hence $r_{B}(m)=[B / m B: A / m]<\infty$ for all maximal ideals of $A$. It follows from [11, Theorem 2.1] that $B$ is $A$-finite.

Now if we assume $I=0$, we have $B \simeq A[[x]] \simeq \Pi_{0}^{\infty} A \subset \bigoplus_{\alpha} A$ for $B$ is a projective $A$-module. To see that $A$ is artinian it suffices to show that for every prime $P$ of $A, A / P$ is a field. By passing to the ring $A / P$ we obtain that $A / P$ is reduced and torsion free and we may apply [3, Corollary 2] to get to a contradiction if $P$ is not maximal. Q.E.D.

The next result extends the preceding to the case in which $A$ is not connected.

THEOREM 3.2. Let $A$ be a noetherian ring, $B$ as in (1) flat and $I \neq 0$. Then $B$ is projective if and only if

1. $c(I)=A e$ with $e$ idempotent.

2. $A(1-e)$ is an artinian ring.

3. $e B$ is Ae-finite.

Proof. By Theorem 2.4, 1 holds. For 2, call $J=c(I)$ and repeat the proof of Theorem 3.1 for $I / J I=0$.

To prove 3 , since $c(I)=A e$, without loss of generality we may assume that $c(I)=A$. Decompose $A=A e_{1} \times A e_{2} \times \cdots \times A e_{n}$ where each $A e_{i}$ is connected and $e_{i}^{2}=e_{i}$. If we tensor (1) with $A e_{i}$ we obtain

$$
0 \rightarrow I e_{i} \rightarrow A e_{i}[[x]] \rightarrow B e_{i} \rightarrow 0 .
$$

Note that $I e_{i} \neq 0$ and so by Theorem $3.1, B e_{i}$ is $A e_{i}$-finite for all $i$. Therefore, $B$ is $A$-finite.

Conversely, let us assume that 1,2 , and 3 hold and tensor (1) with $A / J$ to get $A(1-e)[[x]] \simeq B(1-e)$. Since $A(1-e)$ is artinian, $A(1-e)[[x]]$ is projective as $A(1-e)$-module [2, Theorems 3.3 and 3.4], and hence so is $B(1-e)$. Also $B e=B \otimes_{A} A e$ is flat and finitely presented $A e$-module and therefore it is $A e$-projective [2, Corollary of Proposition 2.2]. From the last two 
statements it follows that $B$ is $A$-projective. Q.E.D.

COROllary 3.3. Let $A$ be a noetherian ring, $B$ as in (1) flat analytic extension of $A$. Assume $c(I)=A$, then $B$ is projective if and only if $B$ is A-finite.

If we put together Theorem 2.4 and Corollary 3.3 we have the following:

THEOREM 3.4. Let $A$ be a noetherian ring and $B$ as in (1). Then $B$ is a flat analytic extension of $A$ if and only if $I$ is projective ideal of $A[[x]]$ and $c(I)$ is generated by an idempotent element of $A$. Moreover, if $c(I)=A$, then $B$ is A-projective if and only if $B$ is a finitely generated flat A-module.

4. Projectivity of analytic extensions. (Case of $\boldsymbol{n}$ indeterminates.)

Let us now consider the question of the projectivity of an analytic algebra arising from an exact sequence

$$
0 \rightarrow I \rightarrow A\left[\left[x_{1}, \cdots, x_{n}\right]\right] \rightarrow B \rightarrow 0,
$$

where $I$ is an $A\left[\left[x_{1}, \cdots, x_{n}\right]\right]$-ideal. (Notice that the question of the projectivity of $I$ treated in the first section is irrelevant for more than one variable.)

For $n>1$, if the ring $A$ is artinian, we cannot infer that $B$ is $A$-artinian from the projectivity of $B$ even if $c(I)=A$, as it is shown in the following simple example.

Example. Let $A$ be any artinian ring and consider the ideal $I=(y)$ in $A[[x, y]]$. Then $B=A[[x]]$ is a flat $A$-module. Moreover, by [2, Theorems 3.3 and 3.4] it is projective, and $B$ is not $A$-finite.

Therefore we need to assume that $A$ is not artinian, or equivalently, since we are dealing with noetherian rings, that Krull $\operatorname{dim} A \geqslant 1$ [6, p. 25].

We will treat first the problem when $A$ is a one dimensional domain. Before dealing with the question we will develop some machinery.

LEMMA 4.1. Let $J=\left(a_{1}, a_{2}, \cdots, a_{n}\right)$ be a finitely generated ideal of a domain $A$. If $J \bar{A}$ is invertible in $\bar{A}$, then there exists a ring $A \subset A^{*} \subset \bar{A}$, such that $A^{*}$ is $A$-finite and $J A^{*}$ is invertible in $A^{*}$.

Proof. Since $J \bar{A}$ is invertible in $\bar{A}$ there exists a finitely generated ideal $L$ of $\bar{A}$ such that $J \bar{A} L=\bar{A} d$. Assume $L=\left(u_{1}, \cdots, u_{n}\right)$ with $u_{i}$ in $\bar{A}$. Then we have equations

$$
\sum_{i, j}^{n} a_{i} u_{j} v_{i j}=d \quad \text { and } \quad a_{i} u_{j}=w_{i j} d
$$

with $w_{i j}$ and $v_{i j} \in \bar{A}$.

Let us define the ring $A^{*}=A\left[d, u_{j}, v_{i j}, w_{i j}\right]$ which is $A$-finite and $A \subset A^{*} \subset \bar{A}$. If $L_{1}$ is the ideal generated by the $u_{i}$ 's in $A^{*}$, then $J A^{*} \cdot L_{1}$ 
$=d A^{*}$ and $J A^{*}$ is invertible in $A^{*}$. Q.E.D.

LEMMA 4.2. Let $A$ be a domain and $f(x)=\Sigma_{i=0}^{\infty} b_{i} x^{i} \in A[[x]]$. Assume that $J=c(f)$ is invertible. Then there is a power series $g(x) \in J^{-1} f$ which has one coefficient equal to the identity.

Proof. By hypothesis we have

$$
1=\sum_{i=1}^{n} k_{i} c_{i} \text { with } \quad c_{i} \in J, \quad k_{i} \in J^{-1} \text {. }
$$

Write $c_{i}=\sum_{i=1}^{s} a_{i j} b_{j}$ with $a_{i j} \in A$. Thus

$$
1=\sum_{i=1}^{n} k_{i} \sum_{j=1}^{s} a_{i j} b_{j}=b_{1} \sum_{i=1}^{n} k_{i} a_{i 1}+\cdots+b_{s} \sum_{i=1}^{n} k_{i} a_{i s} .
$$

Calling $u_{j}=\sum_{i=1}^{n} k_{i} a_{i j} \in J^{-1}$ for $1 \leqslant j \leqslant s$ we have

$$
g(x)=u_{1} x^{s-1} f(x)+\cdots+u_{s-1} x f(x)+u_{s} f(x) \in J^{-1} f
$$

and the sth coefficient of $g$ is $\sum_{i=1}^{s} u_{i} b_{i}=1$. Q.E.D.

Lemma 4.3. Let $A$ be a noetherian ring and consider the following exact sequence of $A$-modules

$$
0 \rightarrow A[[x]] \rightarrow B \rightarrow E \rightarrow 0
$$

with $B$ flat. Then $\operatorname{Tor}_{1}^{A}(A / P, E)=0$ for every prime ideal $P$ if and only if $\operatorname{Tor}_{1}^{A}(A / m, E)=0$ for each maximal ideal $m$.

Proof. The proof is essentially the same as in Lemma 2.3 up to the point when we had that $\operatorname{Tor}_{1}^{A}(A / P, E)$ was divisible by any $0 \neq \bar{a} \in A / P$, and the following exact sequence of $A / P$-modules

$$
0 \rightarrow \operatorname{Tor}_{1}^{A}(A / P, E) \rightarrow A / P[[x]] \rightarrow B / P B \rightarrow E / P E \rightarrow 0 .
$$

Proceeding now with our proof, we have that $A / P[[x]]$ admits a nonzero divisible $A / P$-submodule and this will force $A / P$ to be a field, which is not the case. Q.E.D.

Theorem 4.4. Let $A$ be a noetherian domain of Krull dimension 1. Consider the following exact sequence of A-modules

$$
0 \rightarrow I \stackrel{\lambda}{\longrightarrow} A\left[\left[x_{1}, \cdots, x_{n}\right]\right] \stackrel{\gamma}{\longrightarrow} B \rightarrow 0 .
$$

If $B$ is a projective $A$-module and $I \cap A\left[\left[x_{i}\right]\right] \neq 0$ for at least $n-1 x_{i}$ 's, then $B$ is $A$-finite.

Proof. First we are going to examine the meaning of the conditions:

$$
I \cap A\left[\left[x_{i}\right]\right]=0 \text {, }
$$




$$
I \cap A\left[\left[x_{i}\right]\right] \neq 0 \text {. }
$$

(a) Assume for instance $I \cap A\left[\left[x_{1}\right]\right]=0$. Then $\gamma_{1}$, the restriction of $\gamma$ to $A\left[\left[x_{1}\right]\right]$, is a monomorphism and we have

$$
0 \rightarrow A\left[\left[x_{1}\right]\right] \stackrel{\gamma_{1}}{\longrightarrow} B \rightarrow E \rightarrow 0 \text { exact. }
$$

We claim that $A\left[\left[x_{1}\right]\right]$ is not pure in $B$. If it were so, with $A\left[\left[x_{1}\right]\right]$ and $B$ both flat, $E$ would also be flat. Therefore, by [10, Corollary 3.2.7], proj $\operatorname{dim} E \leqslant 1$, and $A\left[\left[x_{1}\right]\right]$ would be projective. Apply [3, Corollary 2] to get $\Pi_{1}^{\infty} A \subset \bigoplus_{1}^{r} A$ which is a contradiction to the noetherianness of $A$. Thus $\gamma_{1}$ is not pure, and because $A$ is noetherian, this is equivalent to $\operatorname{Tor}_{1}^{A}(A / P, E) \neq$ 0 for some prime ideal $P$ (Proposition 1.1). According to Lemma 4.3 this implies that $\operatorname{Tor}_{1}^{A}(A / m, E) \neq 0$ for some maximal ideal $m$. Tensoring the above sequence with $A / m$ we have the following situation

$$
0 \rightarrow I / m I \rightarrow A /\left.m\left[\left[x_{1}, \cdots, x_{n}\right]\right] \stackrel{A}{\rightarrow}\right|_{B / m B} ^{A / m\left[\left[x_{1}\right]\right]} \theta 0
$$

where $\theta$, the restriction of $\bar{\gamma}$ to $A / m\left[\left[x_{1}\right]\right]$, is not a monomorphism, i.e., there exists $0 \neq \bar{f}\left(x_{1}\right) \in A / m\left[\left[x_{1}\right]\right]$ such that $\bar{\gamma}(\bar{f})=0$. Thus

$$
0 \neq \bar{f}\left(x_{1}\right)=\bar{a}_{0}+\bar{a}_{1} x_{1}+\cdots \in I / m I \text {. }
$$

Let $s$ be least such that $\bar{a}_{s} \neq 0$, and we may assume, by multiplication by an element not in $m$, that $\bar{a}_{s}=1$. Then $\bar{f}\left(x_{1}\right)=x_{1}^{s}+\bar{a}_{s+1} x_{1}^{s+1}+\cdots \in$ $I / m I$ for that maximal ideal $m$, and hence there exists

$$
h\left(x_{1}, \cdots, x_{n}\right)=\sum_{i=0}^{\infty} a_{i} x_{1}^{i}+u\left(x_{1}, \cdots, x_{n}\right) \in I,
$$

where $u\left(x_{1}, \cdots, x_{n}\right) \in m A\left[\left[x_{1}, \cdots, x_{n}\right]\right], a_{i} \in m$ for $1 \leqslant i \leqslant s-1$ and $a_{s} \notin m$.

(b) Assume for instance $I \cap A\left[\left[x_{1}\right]\right] \neq 0$ and let $0 \neq g\left(x_{1}\right) \in I \cap$ $A\left[\left[x_{1}\right]\right]$ and $J=c(g)$. Since $A$ has Krull dimension one, $\bar{A}$ is a Dedekind domain, and thus $J \bar{A}$ is an invertible ideal of $\bar{A}$. Use Lemma 4.1 to obtain $A \subset A^{*} \subset A$ where $A^{*}$ is $A$-finite and $J A^{*}=J^{*}$ is an invertible $A^{*}$-ideal. Applying now Lemma 4.2 we may assume that there exists a power series

$$
f\left(x_{1}\right) \in\left(J^{*}\right)^{-1} g
$$

with some unit coefficient.

If we tensor (3) with $A^{*}$ and we call $I^{*}=I \otimes_{A} A^{*}=I A^{*}$ and $B^{*}=$ $B \otimes_{A} A^{*}$, we obtain 


$$
0 \rightarrow I^{*} \stackrel{\lambda^{*}}{\longrightarrow} \cdot A\left[\left[x_{1}, \cdots, x_{n}\right]\right] \stackrel{\gamma^{*}}{\longrightarrow} B^{*} \rightarrow 0,
$$

for $A\left[\left[x_{1}, \cdots, x_{n}\right]\right] \otimes_{A} A^{*}=A^{*}\left[\left[x_{1}, \cdots, x_{n}\right]\right]$ since $A^{*}$ is finitely presented as $A$-module.

If we call $g^{*}=g \otimes 1^{*} \in I^{*}$, then $\lambda^{*}\left(g^{*}\right)=g 1^{*}$ and $c_{A} *\left(g 1^{*}\right)=$ $J A^{*}=J^{*}$ is an invertible ideal. Now we claim that $\left(J^{*}\right)^{-1} g \subset I^{*}$. In fact, if $0 \neq s \in J^{*}, s\left(J^{*}\right)^{-1} g \subset A^{*} g \subset I^{*}$. Apply $\gamma^{*}$ to get

$$
0=\gamma^{*}\left(s\left(J^{*}\right)^{-1} g\right)=s \gamma^{*}\left(\left(J^{*}\right)^{-1} g\right) \subset B^{*} .
$$

But $B^{*}$ is torsion free, so $\gamma^{*}\left(\left(J^{*}\right)^{-1} g\right)=0$, i.e., $\left(J^{*}\right)^{-1} g \subset I^{*}$ and our claim is proved. This together with (6) implies that $f\left(x_{1}\right) \in I^{*}, f\left(x_{1}\right)$ having one coefficient equal to the identity. Note that the extension $A^{*}$ depends on $x_{1}$. We could repeat the same procedure for all $i$ 's such that (5) holds and obtain extensions $A \subset A_{i}^{*} \subset \bar{A}$ for such $i$ 's. So we actually may consider one extension $A \subset A^{*} \subset \bar{A}$ that works for all $x_{i}$ 's verifying (5). After the change of scalars, we may assume that there exists, for each variable with $I \cap A\left[\left[x_{i}\right]\right]$ $\neq 0$, a power series $f_{i}\left(x_{i}\right) \in I^{*}$ with some coefficient equal to the identity.

We now proceed with our proof proper, considering the two following cases.

First case. Assume $I \cap A\left[\left[x_{i}\right]\right] \neq 0$ for $i=1, \cdots, n$, and let $A^{*}$ and $f_{i}\left(x_{i}\right) \in I$ obtained as in (b). Tensor (3) with $A^{*}$ to get

$$
0 \rightarrow I^{*} \rightarrow A^{*}\left[\left[x_{1}, \cdots, x_{n}\right]\right] \rightarrow B^{*} \rightarrow 0 .
$$

If $M$ is any maximal ideal of $A^{*}$, let us tensor (7) with $A^{*} / M$ to get

$$
0 \rightarrow I^{*} / M I^{*} \rightarrow A^{*} / M\left[\left[x_{1}, \cdots, x_{n}\right]\right] \rightarrow B^{*} / M B^{*} \rightarrow 0,
$$

and

$$
B^{*} / M B^{*}=\frac{A^{*} / M\left[\left[x_{1}, \cdots, x_{n}\right]\right]}{I^{*} / M I^{*}}=\frac{A^{*} / M\left[\left[x_{1}, \cdots, x_{n}\right]\right] /\left(f_{1}^{*}\left(x_{1}\right), \cdots, f_{n}^{*}\left(x_{n}\right)\right)}{I^{*} / M I^{*} /\left(f_{1}^{*}\left(x_{1}\right), \cdots, f_{n}^{*}\left(x_{n}\right)\right)}
$$

a finite $A^{*} / M$-module where $f_{i}^{*}$ are the images of $f_{i}$ in $A^{*} / M\left[\left[x_{i}\right]\right]$. Therefore, $r_{B^{*}}(M)=\left[B^{*} / M B^{*}: A^{*} / M\right]<\infty$ for any maximal ideal $M$ of $A^{*}$, which implies that $B^{*}$ is $A^{*}$-finite [11, Theorem 2.1]. Since $A^{*}$ is $A$-finite, we have that $B^{*}$ is $A$-finite. But $B \subset B^{*}$ because $B$ is $A$-flat, and hence $B$ is $A$-finite.

Second case. Assume $I \cap A\left[\left[x_{i}\right]\right] \neq 0$ for $i=2, \cdots, n$ and $I \cap$ $A\left[\left[x_{1}\right]\right]=0$. We may assume as in (b) that there exist a finite extension $A \subset A^{*} \subset \bar{A}$ and power series $f_{i}\left(x_{i}\right) \in A I^{*}=I^{*}$ having some coefficient equal 
to 1 for $i=2, \cdots, n$. By (a) there is a maximal ideal $m$ and $h\left(x_{1}, \cdots, x_{n}\right)$ $=f_{1}\left(x_{1}\right)+u\left(x_{1}, \cdots, x_{n}\right) \in I$ such that $f_{1}\left(x_{1}\right)$ has one coefficient not in $m$ and $u\left(x_{1}, \cdots, x_{n}\right) \in m A\left[\left[x_{1}, \cdots, x_{n}\right]\right]$. Let $M$ be a maximal ideal of $A^{*}$ such that $M \cap A=m$ and tensor (3) with $A^{*} / M$ to get

$$
0 \rightarrow I^{*} / M I^{*} \rightarrow A^{*} / M\left[\left[x_{1}, \cdots, x_{n}\right]\right] \rightarrow B^{*} / M B^{*} \rightarrow 0 .
$$

The image of $h\left(x_{1}, \cdots, x_{n}\right)$ in $I^{*} / M I^{*}$ is $h^{*}\left(x_{1}, \cdots, x_{n}\right)=f_{1}^{*}\left(x_{1}\right) \neq$ 0. Then

$$
B^{*} / M B^{*}=\frac{A^{*} / M\left[\left[x_{1}, \cdots, x_{n}\right]\right] /\left(f_{1}^{*}\left(x_{1}\right), \cdots, f_{n}^{*}\left(x_{n}\right)\right)}{I^{*} / M I^{*} /\left(f_{1}^{*}\left(x_{1}\right), \cdots, f_{n}^{*}\left(x_{n}\right)\right)}
$$

is a finite $A^{*} / M$-module where $f_{i}^{*}\left(x_{i}\right)$ are the images of $f_{i}\left(x_{i}\right)$ in $A^{*} / M\left[\left[x_{1}, \cdots, x_{n}\right]\right]$ for $i \geqslant 2$. Therefore, $r_{B^{*}}(M)=\left[B^{*} / M B^{*}: A^{*} / M\right]<\infty$ for some maximal $M$; it follows now from Proposition 1.2 that $r_{B^{*}}(M)$ is finite for any maximal ideal $M$ of $A^{*}$. Thus $B^{*}$ is $A^{*}$-finite and we conclude as in the first case, that $B$ is $A$-finite. Q.E.D.

Before we consider the case when $\operatorname{Krull} \operatorname{dim} A \geqslant 1$, we will prove the following.

Lemma 4.5. Let $(A, m)$ be a local noetherian domain with Krull $\operatorname{dim} A>1$. If $J \subset P$ for every prime $P \neq 0$, then $J=0$.

Proof. Assume that $J \neq 0$, and let $P_{1}, \cdots, P_{r}$ be the minimal primes over $J$. Since Krull $\operatorname{dim} A>1$, there exists a prime ideal $0 \neq P \varsubsetneqq m$. If $m$ $\subset \bigcup_{i=1}^{r} P_{i}$, then $m$ would be the only minimal prime over $J$. We then would have $m \subset P$. Hence there exists

$$
a \in m-\bigcup_{i=1}^{r} P_{i}
$$

Let $Q_{1}, \cdots, Q_{n}$ be the minimal primes over the ideal generated by $a$. It follows from the Principal Ideal Theorem [4, Theorem 152] that the height of the $Q_{i}$ 's is one, which implies that they are minimal over $J$, and $a \in$ $\bigcup_{i=1}^{r} P_{i}$, contradicting (8). Q.E.D.

Theorem 4.6. Let $A$ be a noetherian domain with $\operatorname{Krull} \operatorname{dim} A \geqslant 1$. Let $B$, as in (3), be a flat $A$-module and $I \cap A\left[\left[x_{i}\right]\right] \neq 0$ for at least $n-1$ $x_{i}$ 's. Then $B$ is A-projective if and only if it is $A$-finite.

Proof. The case when $\operatorname{dim} A=1$ is considered in Theorem 4.4, so we may assume $\operatorname{Krull} \operatorname{dim} A>1$. For each $i$ such that $I \cap A\left[\left[x_{i}\right]\right] \neq 0$, let $0 \neq f_{i}\left(x_{i}\right) \in I$. Set $J_{i}=c\left(f_{i}\right)$ and let $J$ be the product of the $J_{i}$ 's for such $i$ 's. If $J=A$, take any prime $P$ such that $\operatorname{dim} A / P=1$. It follows that the 
image of $f_{i}\left(x_{i}\right)$ in $I / P I$ is different from zero for those $i$ 's such that $I \cap$ $A\left[\left[x_{i}\right]\right] \neq 0$. We are under the hypothesis of Theorem 4.4 and therefore $B / P B$ is $A / P$-finite. But $B / m B \simeq(B / P B) /(m B / P B)$ is $A / P$-finite if $m$ is a maximal ideal of $A$ containing $P$, which implies that $B$ is $A$-finite by Proposition 1.2 and [11, Theorem 2.1].

If $J \neq A$, assume that there is a prime ideal $P$ such that

$$
\text { Krull } \operatorname{dim} A / P=1 \text { and } J \not \subset P .
$$

Therefore, the image of $f_{i}\left(x_{i}\right)$ in $I / P I$ is not zero for those $i$ 's such that $I \cap$ $A\left[\left[x_{i}\right]\right] \neq 0$ and we apply, as before, Theorem 4.4 to obtain that $B$ is $A$-finite.

So we just have to find $P$ satisfying (9). Assume, on the contrary, that for every prime $P$ with $\mathrm{Krull} \operatorname{dim} A / P=1$ we have $J \subset P$. One may assume, without loss of generality, that $J \subset P$ for every nonmaximal ideal $P \neq 0$. (Otherwise pass to the ring $A / P$ with $P$ maximal with the property that $J \not \subset P$.) Let $m$ be a maximal ideal containing $J$. By localizing at $m$ we may assume that $A$ is local with maximal ideal $m$. Since $A$ is a domain, $J$ will remain different from zero after localizing, and then we may apply Lemma 4.5 to get a contradiction if $\mathrm{Krull} \operatorname{dim} A>1$.

The converse is trivial by [2, Proposition 2.2]. Q.E.D.

To avoid the cumbersome hypothesis on $I$ of Theorem 4.6 we consider the case of a local ring.

Theorem 4.7. Consider the exact sequence (3) with $(A, m)$ a local noetherian ring with $\mathrm{Krull} \operatorname{dim} A \geqslant 1$ and $B$ flat $A$-module. Then $B$ is a projective A-module if and only if it is $A$-finite.

Proof. Assume first that $A$ is a domain of Krull dimension one and let

$$
I \cap A\left[\left[x_{i}\right]\right]=0 \text { for } i=1, \cdots, r
$$

and

$$
I \cap A\left[\left[x_{i}\right]\right] \neq 0 \text { for } i=r+1, \cdots, n .
$$

Therefore for $i=1, \cdots, r$ we have power series $h_{i}\left(x_{1}, \cdots, x_{n}\right)=f_{i}\left(x_{i}\right)+$ $u_{i}\left(x_{1}, \cdots, x_{n}\right)$ with $u_{i}\left(x_{1}, \cdots, x_{n}\right) \in m A\left[\left[x_{1}, \cdots, x_{n}\right]\right]$ and $f_{i}\left(x_{i}\right)$ having one coefficient not in $m$, by (a) of Theorem 4.4. As for $i=r+1$, $\cdots, n$ we have $A \subset A^{*} \subset \bar{A}$ and $f_{i}\left(x_{i}\right) \in I^{*}$, where each $f_{i}$ has one coefficient equal to 1 by (b) of the same theorem.

Let $M$ be a maximal ideal in $A^{*}$ such that $M \cap A=m$. Then for $i=1, \cdots, n, f_{i}^{*}\left(x_{i}\right)$, the image of $f_{i}$ in $I^{*} / M I^{*}$, has one coefficient equal to 1 . 
It follows as in the second case of Theorem 4.4 that $B^{*}$ is $A^{*}$-finite, and therefore $B$ is $A$-finite.

Now, if $A$ is not one dimensional, we can find a prime ideal $P$ such that $\operatorname{dim} A / P=1$. Thus we obtain that $B / P B$ is $A / P$-finite and hence $B / m B$ is $A / P$-finite, if $m$ is a maximal ideal of $A$ containing $P$, which implies $r_{B}(m)=[B / m B: A / m]<\infty$, and $B$ is again $A$-finite by Proposition 1.2 and [11, Theorem 2.1].

The converse is trivial by [2, Proposition 2.2]. Q.E.D.

It is likely that Theorem 4.7 holds in all cases, not just for local rings.

5. Finiteness of $I$ when $A[[x]] / I$ is flat. The finiteness of $I$ when. $A[x] / I$ is a flat extension of $A$-for $A$ not necessarily noetherian-has been studied by several authors, especially in [7], [8], [9] and [13]. So far, related problems for flat analytic extensions have not been treated. In this section we will present two minor contributions to the subject, for local rings.

Lemma 5.1. Let $(A, m)$ be a local ring, and $I \neq 0$ an ideal of $A[[x]]$. Consider the following exact sequence of A-modules

$$
0 \rightarrow I \rightarrow A[[x]] \stackrel{\gamma}{\longrightarrow} B \rightarrow 0
$$

with $B$ flat. If there exists $0 \neq f(x) \in I$ such that $J=c(f)$ is finitely generated, then $c(I)=A$.

Proof. Let us reason by induction on the number of generators of $J$, assuming first $J=(d)$; then $f(x)=d\left(b_{0}+b_{1} x+\cdots\right)=d g(x)$ and $(d)=$ $c(f)=d c(g)$.

If $c(g) \neq A$, by Nakayama's lemma $d=0$. Therefore $c(g)=A$, and $g(x)$ has one coefficient that is a unit. Since $f \in I$ one has $d \gamma(g)=\gamma(d g)=$ $\gamma(f)=0$. So $\gamma(g) \in\left(0:_{B} d\right)=\{b \in B \mid d b=0\}$ and since $B$ is flat $\left(0:_{B} d\right)$ $=\left(0:_{A} d\right) B \subset m B$. Thus $\gamma(g)=\sum_{i=1}^{s} r_{i} \gamma\left(h_{i}\right)$ with $r_{i} \in m, h_{i} \in A[[x]], 1 \leqslant$ $i \leqslant s$.

If $h=g-\sum_{i=1}^{s} r_{i} h_{i}$, then $h \in I$ and $c(h)=A$.

Assume now $J=\left(d_{1}, \cdots, d_{n}\right), n$ least, and call $L=\left(d_{1}, \cdots, d_{n-1}\right)$. Tensor (10) with $A / L$ to get

$$
0 \rightarrow I / L I \rightarrow A / L[[x]] \rightarrow B / L B \rightarrow 0 \text {. }
$$

Since $J / L=c_{A / L}(\bar{f})=\left(d_{n}\right)$, where $\bar{f}$ is the image of $f$ in $A / L[[x]]$, one has, by the first part and by Lemma 2.2, that there exists $h=\sum_{i=0}^{\infty} c_{i} x^{i} \in I$ with $\bar{c}_{n}=\overline{1}$ in $A / L$. Thus

$$
c_{n}-1 \in L=\left(d_{1}, \cdots, d_{n-1}\right) .
$$


Note that if $d_{i} \notin m$ for some $i$, then $c(I)=A$ and there is nothing to prove. So we may assume $d_{i} \in m$ for all $i$, and it follows from (11) that $c_{n}$ is invertible. Hence $c(h)=1$. Q.E.D.

LEMMA 5.2. Let $A$ be any ring and let $E, F$ be A-modules such that $E$ is pure in $F$, and $F$ free. Assume that $E \subset J F$, where $J$ is the Jacobson radical of $A$. Then $E=0$.

Proof. If $e \in E \subset F$, and $\left\{f_{i}\right\}_{i \in I}$ is a basis of $F$, then $e=\sum_{i=1}^{n} a_{i} f_{i}$, with $a_{i} \in A$. But since $E$ is pure in $F$ there exists $e_{k} \in E$ such that $e=$ $\sum_{k=1}^{n} a_{i} e_{k}$. As $e_{k} \in E \subset F, e_{k}=\sum_{i=1}^{s} t_{k i} f_{i}$ with $t_{k i} \in J$. Thus

$$
e=\sum_{k=1}^{n} a_{k} \sum_{i=1}^{s} t_{k i} f_{i}=\sum_{i=1}^{n} a_{i} f_{i}
$$

and therefore $a_{i}=\sum_{k=1}^{n} a_{k} t_{k i}, i=1, \cdots, s$.

Call $m=\left(a_{1}, \cdots, a_{n}\right)$ and let $L$ be the ideal generated by the $t_{k i}$ 's, $1 \leqslant i \leqslant s, 1 \leqslant k \leqslant n$. Clearly $L \subset J$ and $M=M L$, so we may apply Nakayama's lemma to obtain $M=0$, i.e., $e=0$. Q.E.D.

Lemma 5.3. Let $(A, m)$ be a local ring and let $B$ as in (10) be a flat analytic extension of $A$. Assume that $x^{n} \in I$ and that all first $n$ coefficients of $g$ are in $m$ for every $g \in I$. Then $I=\left(x^{n}\right)$.

Proof. The sequence $0 \rightarrow I /\left(x^{n}\right) \rightarrow A[[x]] /\left(x^{n}\right)$ is pure since $B$ is flat. Also $A[[x]] /\left(x^{n}\right)$ is a free $A$-module, and if $g \in I$, its image $\bar{g}$ in $I /\left(x^{n}\right)$ verifies

$$
\bar{g}(x)=\sum_{i=0}^{\infty} \bar{a}_{i} x^{i}=\sum_{i=0}^{n-1} \bar{a}_{i} x^{i} \in m A[[x]] /\left(x^{n}\right)
$$

since by hypothesis $a_{i} \in m$ for all $i<n$. Thus apply Lemma 5.2 to the situation $I /\left(x^{n}\right) \subset m \cdot A[[x]] /\left(x^{n}\right)$ to obtain $I=\left(x^{n}\right)$. Q.E.D.

THEOREM 5.4. Let $(A, m)$ be a local ring and $B$ as in (1) be a flat analytic extension of $A$. Assume $I$ is finitely generated and $c(I)=A$. Then $I$ is principal.

Proof. It is enough to show that there is a finitely generated ideal $J \subset$ $m$ such that $I / J I$ is a principal $A / J[[x]]$-ideal: Because if it were so, $I / J I=$ $\bar{g}(x) A / J[[x]]$ and hence $I=J I+(g) A[[x]]$ with $g \in I$. Then by Nakayama's lemma, $1=(g)$.

Let us then find $J$ as above. By Lemma 2.2 there exists $h \in I$ such that $h=\sum_{i=0}^{\infty} b_{i} x^{i}$ and $b_{n}=1$. Take $h$ with $n$ minimal and let $J=$ $\left(b_{0}, \cdots, b_{n-1}\right) \subset m$. Tensor (10) with $A / J$ to get 


$$
0 \rightarrow I / J I \rightarrow A / J[[x]] \rightarrow B / J B \rightarrow 0 .
$$

The image of $h$ in $I / J I$ is $\bar{h}=x^{n}+\bar{b}_{n+1} x^{n+1}+\cdots=x^{n} \bar{f}(x)$ with $\bar{f}(x)=$ $1+b_{n+1} x+\cdots$ invertible in $A / J[[x]]$. Therefore $x^{n} \in I / J I$. It follows from Lemma 5.3 that $I / J I=\left(x^{n}\right) A / J[[x]]$ and the theorem is proved. Q.E.D.

COROllary 5.5. Let $(A, m)$ be a local ring, I a finitely generated $A[[x]]$-ideal, and $B$ as in (10) a flat analytic extension of $A$. If there exists $f \in I$ such that $c(f)$ is finitely generated, then $I$ is principal.

Proof. Apply Lemma 5.1 to get $c(I)=A$. Q.E.D.

An example where $c(I)$ is not finitely generated can be obtained as follows. Let $A=k\left\{t_{1}, t_{2}, \cdots, t_{h}, \cdots\right\}$ be the ring of power series in $t_{1}, \cdots, t_{h}, \cdots$ where only fiinitely many indeterminates occur in each series, i.e.,

$$
A=\bigcup_{h}^{\infty} k\left[\left[t_{1}, \cdots, t_{h}\right]\right]=\bigcup_{h}^{\infty} A_{h} .
$$

Since $A$ is the direct limit of the coherent rings $A_{h}$, and all $A_{h}$ 's are flat $A_{j}$-modules if $j \leqslant h$, it follows that $A$ is also coherent. If we set $f=$ $t_{1} x+t_{2} x^{2}+\cdots \in A[[x]]$, and call $I=(f)$, we have

$$
0 \rightarrow I \rightarrow A[[x]] \rightarrow B \rightarrow 0 .
$$

Notice that $A$ is local and $c(I)$ coincides with its maximal ideal, which is not finitely generated. We claim here that $B$ is a flat $A$-module. Let us consider the sequence $0 \rightarrow A[[x]] \stackrel{f}{\rightarrow} A[[x]]$ and tensor it with $A / J$, where $J$ is a finitely generated ideal to get

$$
\operatorname{Tor}_{1}^{A}(B, A / J) \rightarrow A / J[[x]] \stackrel{\bar{f}}{\longrightarrow} A / J[[x]] .
$$

Since $A$ is coherent, to see that $\operatorname{Tor}_{1}^{A}(B, A / J)=0$ it is enough to show that $\bar{f}$ is a non-zero-divisor of $A / J[[x]]$. Assume that $\bar{f} \bar{g}=0$ for $0 \neq$ $\bar{g}=\Sigma_{i=1}^{\infty} \bar{b}_{i} x^{i} \in A / J[[x]]$. (Note that we may already assume that $\bar{g}$ has no constant term, because otherwise we take $x \bar{g}$.)

Since $J$ is a finitely generated ideal of $A, J$ may be written as $J=J_{0} A$, where $J_{0}$ is an ideal of $k\left[\left[t_{1}, \cdots, t_{n}\right]\right]$ for some integer $n$, and it follows that $A / J=\left(A_{n} / J_{0}\right)\left\{t_{n+1}, t_{n+2}, \cdots\right\}$.

The $(n+p+1)$ th coefficient of $\bar{f} \bar{g}=0$ translates into

$$
t_{n+1} \bar{b}_{p}+\bar{t}_{n} \bar{b}_{p+1}+\cdots+\bar{t}_{2} \bar{b}_{n+p-1}+\bar{t}_{1} \bar{b}_{n+p}=0,
$$

where $\bar{b}_{p}$ is the first nonzero coefficient of $\bar{g}$.

Since only finitely many indeterminates are involved in each $\bar{b}_{i} \neq 0$ with 
$p \leqslant i \leqslant n+p$, let $m$ be the largest index such that $\bar{t}_{m}$ occurs in those $\bar{b}_{i}$ 's.

From (12) we can see that $m \geqslant n+1$, so actually

$$
\bar{t}_{i}=t_{i} \neq 0 \text { for } i \geqslant m \text {. }
$$

Let us define the ring homomorphism

$$
\lambda: A / J[[x]] \rightarrow\left(A_{n} / J_{0}\right)\left[\left[t_{n+1}, \cdots, t_{m}\right]\right][[x]]
$$

such that

$$
\begin{array}{ll}
\lambda\left(t_{i}\right)=0 & \text { if } i>m, \\
\lambda\left(\overline{t_{i}}\right)=\bar{t}_{i} & \text { if } i \leqslant m .
\end{array}
$$

By (13) both $\lambda(\bar{f})$ and $\lambda(\bar{g})$ are different from zero, and

$$
\lambda(\bar{f})=\bar{t}_{1} x+\bar{t}_{2} x^{2}+\cdots+\bar{t}_{m-1} x^{m-1}+t_{m} x^{m}
$$

has one coefficient, precisely $t_{m}$, which is a non-zero-divisor.

Now the ring we are working in is $A_{n} / J_{0}\left[\left[t_{n+1}, \cdots, t_{m}\right]\right]$ which is noetherian, and therefore we may apply Lemma 2.1 to get $\lambda(\bar{g})=0$, a contradiction.

THEOREM 5.6. Let $(A, m)$ be a local ring of Krull dimension zero, and $B$ as in (10) a flat analytic extension of $A$. If $c(I)=A$, then $I$ is principal.

Proof. By Lemma 2.2 there exists $f=\sum_{i=0}^{\infty} a_{i} x^{i} \in I$ with $a_{n}=1$. Pick $f \in I$ with $n$ minimal. Let $J$ be the ideal of $A$ generated by $a_{0}, a_{1}, \cdots$, $a_{n-1}$ and tensor (10) with $A / J$ to get $0 \rightarrow I / J I \rightarrow A / J[[x]] \rightarrow B / J B \rightarrow 0$, and the image of $f$ in $I / J I$ is

$$
\bar{f}=x^{n}+\bar{a}_{n+1} x^{n+1}+\cdots=x^{n} \bar{g}
$$

with $\bar{g}=1+\bar{a}_{n+1} x+\cdots$ invertible in $A / J[[x]]$. Therefore $x^{n} \in I / J I$. It follows from Lemma 5.3 that $I / J I=\left(x^{n}\right) A / J[[x]]$ and then $I=(f)+$ $J A[[x]] \cap I$, where $\bar{f}=x^{n}$. But $J \cdot A[[x]] \cap I=J I$ since $B$ is $A$-flat, thus

$$
I=(f)+J I=(f)+J^{2} I=\cdots=(f)+J^{r} I=\cdots .
$$

But $J^{r}=0$ for some $r$, since $A$ is local and 0 -dimensional. Hence $I=(f)$, i.e., it is principal. Q.E.D.

COROLlaRY 5.7. Let $(A, m)$ be a local ring of Krull dimension zero, and $B$, as in (10), a flat analytic extension of $A$. If there exists $f \in I$ such that $c(f)$ is finitely generated, then $I$ is principal.

Proof. Apply Lemma 5.1 to get $c(I)=A$. Q.E.D. 


\section{BIBLIOGRAPHY}

1. N. Bourbaki, Eléments de mathématique. Algèbre commutative. Chaps. I-VII, Actualités Sci. Indust., nos. 1290, 1293, 1308, 1314, Hermann, Paris, 1961-1965. MR 30 \#2027; 33 \#2660; 36 \#146; 41 \#5339.

2. S. Chase, Direct product of modules, Trans. Amer. Math. Soc. 97 (1960), 457-473. MR $22 \# 11017$.

3. - A remark on direct products of modules, Proc. Amer. Math. Soc. 13 (1962), 214-221. MR 25 \#1197.

4. I. Kaplansky, Commutative rings, Allyn and Bacon, Boston, Mass., 1970. MR 40 $\# 7234$.

5. - Projective modules, Ann. of Math (2) 68 (1958), 372-377. MR 20 \#6453.

6. M. Nagata, Local rings, Interscience Tracts in Pure and Appl. Math., no. 13, Interscience, New York, 1962. MR 27 \#5790.

7. - Flatness of an extension of a commutative ring, J. Math. Kyoto Univ. 9 (1969), 439-448. MR 41 \#191.

8. J. Ohm and D. E. Rush, The finiteness of $I$ when $R[x] / I$ is flat, Trans. Amer. Math. Soc. 171 (1972), 377-408. MR 46 \#5303.

9. J. Ohm and W. Heinzer, The finiteness of $I$ when $R[x] / I$ is $R$-flat. II, Proc. Amer. Math. Soc. 35 (1972), 1-8. MR 46 \#5304.

10. M. Raynaud and L. Gruson, Critères de platitude et de projectivité. Techniques de "platification"d'un module, Invent. Math. 13 (1971), 1-89. MR 46 \#7219.

11. W. V. Vasconcelos, On projective modules of finite rank, Proc. Amer. Math. Soc. 22 (1969), 430-433. MR 39 \#4134.

12. - Simple flat extensions, J. Algebra 16 (1970), 105-107. MR 42 \#252.

13. - Simple flat extensions. II, Math. Z. 129 (1972), 57-161. VENEZUELA

DEPARTAMENTO DE MATEMÁTICAS, UNIVERSIDAD DE ORIENTE, CUMANÁ, 